\title{
A Perceptual-Motor Deficit Predicts Social and Communicative Impairments in Individuals With Autism Spectrum Disorders
}

\author{
Sally A. Linkenauger, Matthew D. Lerner, Verónica C. Ramenzoni, and Dennis R. Proffitt
}

\begin{abstract}
Individuals with autism spectrum disorders (ASDs) have known impairments in social and motor skills. Identifying putative underlying mechanisms of these impairments could lead to improved understanding of the etiology of core social/communicative deficits in ASDs, and identification of novel intervention targets. The ability to perceptually integrate one's physical capacities with one's environment (affordance perception) may be such a mechanism. This ability has been theorized to be impaired in ASDs, but this question has never been directly tested. Crucially, affordance perception has shown to be amenable to learning; thus, if it is implicated in deficits in ASDs, it may be a valuable unexplored intervention target. The present study compared affordance perception in adolescents and adults with ASDs to typically developing (TD) controls. Two groups of individuals (adolescents and adults) with ASDs and age-matched TD controls completed well-established action capability estimation tasks (reachability, graspability, and aperture passability). Their caregivers completed a measure of their lifetime social/communicative deficits. Compared with controls, individuals with ASDs showed unprecedented gross impairments in relating information about their bodies' action capabilities to visual information specifying the environment. The magnitude of these deficits strongly predicted the magnitude of social/communicative impairments in individuals with ASDs. Thus, social/communicative impairments in ASDs may derive, at least in part, from deficits in basic perceptual-motor processes (e.g. action capability estimation). Such deficits may impair the ability to maintain and calibrate the relationship between oneself and one's social and physical environments, and present fruitful, novel, and unexplored target for intervention. Autism Res 2012, 5: 352-362. () 2012 International Society for Autism Research, Wiley Periodicals, Inc.
\end{abstract}

Keywords: autism spectrum disorders; social deficits; perception; perceptual-motor integration; action capability estimation; affordance perception

Since their earliest descriptions, individuals with autism spectrum disorders (ASDs) have been depicted as exhibiting characteristic social/communicative deficits and general motor problems (Asperger, 1944, as cited in Wing, 1981; Forti et al., 2011; Ming, Brimacombe, \& Wagner, 2007). While it has been speculated that both motor and social/communicative deficits may originate from a common etiology (Baranek, Parham, \& Bodfish, 2005), such speculation has not been empirically tested. Herein, we assessed the hypothesis that a very basic perceptualmotor integration deficit may be present in ASD populations, and that this deficit may underlie the more commonly addressed social and motor issues present in individuals with ASDs.

Beginning in early development, people and other animals actively explore their surroundings and, in so doing, learn the relationship between their actions and the optically specified effects that these actions produce in the environment (Gibson, 1966, 1979). The ability to calibrate actions with their perceptual outcomes is commonly referred to as affordance perception and requires perceptual-motor integration. Not only does this calibration allow individuals to anticipate the consequences of their own movements in visual environments, but it also semantically grounds visual information to the organism and its actions. Perceptual-motor integration is fundamental to everyday tasks that require interacting with the environment (e.g. walking through doorways, and deciding when to cross a street; Adolph \& Berger, 2006; Konczak, Meeuwsen, \& Cress, 1992; Mark, 1987; Mark et al., 1990; Oudejans, Michaels, Bakker, \& Dolné, 1996; Stoffregen, Yang, \& Bardy, 2005; Warren, 1984; Warren \& Whang, 1987) and the agents that populate it (e.g. Chang, Wade, \& Stoffregen, 2009; Ramenzoni, Davis, Riley, Shockley, \& Baker, 2011). Affordances perception is thought to be fundamental to the success of social interactions. Humans' ability to understand and predict the movements of others in social contexts (Marsh,

From the Perception and Action in Virtual Environments, Max Planck Institute for Biological Cybernetics, Tübingen, Germany (S.A.L.); Department of Psychology, University of Virginia, Charlottesville, Virginia, USA (M.D.L., D.R.P.); Communication Before Language, Max Planck Institute for Psycholinguistics, Nijmegen, Netherlands (V.C.R.)

Grant sponsor: Jefferson Scholars Foundation; Grant number: James H. and Elizabeth W. Wright Endowed Fellowship (Matthew D. Lerner).

Grant sponsor: National Institute of Health; Grant number: RO1MH075781 (Dennis R. Proffitt).

Received December 28, 2011; accepted for publication July 4, 2012

Address for correspondence and reprints: Sally Linkenauger, Cognitive and Computational Psychophysics Department, Max Planck Institute for Biological Cybernetics, Postfach 21 69, D-72012 Tübingen. E-mail: sally.linkenauger@tuebingen.mpg.de

Published online 7 September 2012 in Wiley Online Library (wileyonlinelibrary.com)

DOI: $10.1002 /$ aur. 1248

(C) 2012 International Society for Autism Research, Wiley Periodicals, Inc. 
Richardson, \& Schmidt, 2009; Ramenzoni, Riley, Shockley, \& Davis, 2008a, 2008b) and to engage in ongoing interactions (Davis, Riley, Shockley, \& Cummins-Sebree, 2010; Richardson, Marsh, \& Baron, 2007) depends to large extent on it.

Because affordance perception contributes to successful performance of many motor and nonverbal social capabilities, it is possible that impairments in basic affordance perception may underlie both the motor problems and the social/communicative deficits evident in persons with ASD. Similar to what is known about developmental dyspraxia (Dewey, 1995; Dewey \& Kaplan, 1992), difficulties in affordance perception stemming from perceptualmotor integration deficits may yield downstream difficulties in communication and interaction abilities. Although the perception of affordances has not yet been empirically investigated with respect to ASDs, people with ASDs have specifically reported difficulty in affordance perception as well as impairment in tasks that require perceptual-motor integration (Attwood, 2008). For example, many individuals with ASDs report problems with walking up stairs, as it requires the motor system to utilize visual information to specify how high to lift up ones foot to ascend the stair. More recently, researchers have found that many of these motor deficits are attributable to deficits in motor planning rather than execution, which would be consistent with an affordance account (Forti et al., 2011). Similarly, deficits in motor skills in individuals with ASDs have been found to be related to sensitivity to biological motion and the ability to use visual information to maintain posture (Price, Shiffrar, \& Kerns, 2012). Such motor skill deficits may directly yield difficulties in elements of social interaction; for instance, de Marchena and Eigsti (2010) found that youth with ASD displayed poorer synchrony between gestures and speech during a narrative task, with degree of synchrony predicting communicative quality. Overall, current research indicates a pattern of motor deficits specific to ASDs, with researchers strongly suggesting that these deficits may emerge from a profound underlying perceptual-motor integration problem (Whyatt \& Craig, in press).

While perceptual-motor integration is typically thought of as allowing individuals to determine their own action capabilities in their environment, there is also a wealth of research supporting the notion that perceivers recruit their own perceptual-motor processes to simulate and, thereby, understand the actions of others (Blakemore \& Decety, 2001; Fabbri-Destro \& Rizzolatti, 2008). Consequently, deficits in perceptual-motor integration could be the reason individuals with ASDs have often been reported to have difficulty identifying and predicting the outcome of other's actions (Boria et al., 2009; Zalla, Labruyere, Clement, \& Georgieff, 2010). While such results have not always been found (Carpenter, Penning- ton, \& Rogers, 2001; de C. Hamilton, Brindley, \& Frith, 2007; Falck-Ytter, 2010), such inconsistency highlights the importance of identifying basic perceptual processes that may underlie difficulty in predicting others' actions. Additionally, perceptual-motor integration allows individuals to coordinate their actions with those of others (Ramenzoni et al., 2008a). Neurotypical individuals almost automatically adjust their own movements to coordinate with those with whom they are interacting (Boker et al., 2011; Ramenzoni et al., 2011), whereas individuals with autism do not show this type of reactivity to the actions of others, which also supports a perceptualmotor account (Marsh et al., 2009). Therefore, perceptual-motor integration is central to not only acting in the environment but also acting in conjunction with others, which is the basis for social and communicative abilities. If those with ASDs are impaired in perceptualmotor integration, it could likely cause deficits in other social-cognitive domains.

In concordance with this condition, a large body of research supports the notion that individuals with ASDs have deficits in interpreting human biological motion from point light displays. Typically developing (TD) individuals are strikingly proficient at identifying and interpreting human movements from displays featuring only lighted dots on the key joints of a human actor starting from 2 days after birth (Simion, Regolin, \& Bulf, 2008). TD individuals are quite accurate in their interpretation of the action being performed as well more subtle information such as gender, body weight, arousal level, and even emotional valence of the individual depicted from only the relative motion of point light markers on the joints of an individual's body (see Kaiser \& Shiffrar, 2009, for a review). Individuals are better at determining the actions in their own point light displays than those of others even from the third-person perspective. Additionally, the ability to process the point light displays decreases as a function of the discrepancy between the motion of the observer's body and the motion of the point light display, suggesting that the ability to interpret these point light displays is likely due to the capacity to simulate the motion in the display with one's own motor system (Parkinson, Springer, \& Prinz, 2011; Springer et al., 2011). This being the case, to identify the action in the point light display, individuals must integrate the visual information specifying the action to their motor system in a form of perceptual motor integration. In correspondence with perceptual-motor deficit idea, some evidence suggests that individuals with ASDs have difficulty interpreting these displays. Children with ASDs are impaired in determining the difference biological motion versus scrambled motion in comparison with aged-matched TD children (Blake, Turner, Smoski, Pozdol, \& Stone, 2003); however, see Moore, Hobson, and Lee (1997) for opposing conclusions from a different experimental design. 
Although adults with ASDs appear to improve in their ability to discriminate biological motion from nonbiological motion from point light displays (Murphy, Brady, Fitzgerald, \& Troje, 2009; Rondan \& Deruelle, 2005), adults with ASDs are still inferior to TD individuals in identifying other aspects from point light displays such as emotional state and naturalness of the movement (Cook, Saygin, Swain, \& Blakemore, 2009; Hubert et al., 2007). Additionally, neural imaging studies have revealed that individuals with ASDs use different neural mechanisms when interpreting biological motion, suggesting that perhaps a compensatory mechanism allows for the amelioration of the deficit (Koldewyn, Whitney, \& Rivera, 2011; McKay et al., 2012). Specifically, individuals with ASDs show less activation in neural areas associated with their own actions than TD individuals when performing these tasks (Freitag et al., 2008). Consequently, individuals with ASD could bypass perceptual-motor integration using simple visual recognition to perform these tasks to compensate for the perceptual-motor integration abilities used by TD individuals.

To test directly for a perceptual motor integration deficit, we had adolescents with ASDs perform affordance perception tasks that require perceptual-motor integration. Adolescents were selected for this initial exploration of this phenomenon for several reasons. First, we wanted to ensure that they had reached an age of high diagnostic stability. Second, we wanted to increase the likelihood of comprehension of tasks that have been well validated in young adult samples. Third, by adolescence, youth might otherwise be expected to develop compensatory capacities, thus providing a strict test of the hypothesis that basic affordance perceptions are impaired in individuals with ASDs. Using well-developed paradigms (Ishak, Adolph, \& Lin, 2008; Warren \& Whang, 1987), we asked these adolescents to estimate whether they could perform simple actions without overt feedback. Specifically, they were asked to estimate the extent of their grasping ability, the maximum extent of their reach, and the largest aperture through which their hands could pass. These tasks require that individuals be able to relate visual information to the action capabilities of their body in the absence of motor feedback.

We chose these tasks for several reasons. First, in terms of hand and arm movements, several studies have shown that children with ASDs are just as accurate with their movement profiles as TD children, only differing by a slight increase in the spatial and temporal variability of movement onset (Forti et al., 2011; Glazebrook, Elliott, \& Lyons, 2006). Second, ASD individuals have intact or even superior abilities in estimation tasks, including those involving vision, such as in size and distance estimation tasks (both verbal and visual matching), visual discrimination tasks, detecting embedded figures, and reproducing visual stimuli by hand (Ashwin, Ashwin, Rhydderch,
Howells, \& Baron-Cohen, 2009; Souliéres et al., 2010). Indeed, in many cases, individuals with ASD demonstrate superior awareness of the relative position of their limbs in space and can use this information in learning new actions (Haswell, Izawa, Dowell, Mostofsky, \& Shadmehr, 2009; Mottron, Dawson, Souliéres, Hubert, \& Burack, 2006). Third, motor planning abilities have shown to be intact in other clinical populations, such as attention deficit/hyperactivity disorder (Klimkeit, Mattingley, Sheppard, Lee, \& Bradshaw, 2005), that might otherwise be comorbid with ASDs (Sinzig, Walter, \& Doepfner, 2009). Fourth, affordance perception is typically not associated with creativity/imagination but rather being able to integrate visual information specifying the environment and the action abilities of the body (Gibson, 1979). Thus, if deficits were found on these tasks, it would be unlikely that they are attributable to problems with general motor or visual estimation abilities, clinical comorbidities, or limitations in imaginative ability; rather, such deficits would support the presence of a specific deficit in perceiving one's own action capabilities. Finally, these tasks (and affordance perception in general) are similar to those that have demonstrated learning effects (Adolph, 1997; Fajen, 2005). Thus, if deficits were found, they would present a promising novel target for intervention to address core deficits in ASDs.

\section{Experiment 1: Affordance Perception and Adolescents With ASDs}

Adolescents with and without ASDs performed several tasks that require perceptual-motor integration. Specifically, we assessed participants' accuracy in their ability to perceive affordances. If individuals with ASDs show greater errors than individuals without ASDs, it is likely that perceptual-motor integration is impaired in those with ASDs.

\section{Methods}

Participants. Twelve male adolescents (age 9-13, $\left.M_{\text {age }}=11.08\right)$ with high-functioning ASDs and 12 agematched TD male adolescents (age 9-13, $M_{\text {age }}=11.08$ ) participated in the experiment after both the participant and their parent gave informed consent. None of the participants had any significant hearing loss, visual impairment, or major physical disability. Documented diagnoses for the all adolescents with ASDs were provided by area clinicians and confirmed for all but one using the autism diagnostic observation schedule (ADOS; Lord, Rutter, DiLavore, \& Risi, 1999) in a separate study $[M=12$, standard deviation $(S D)=4 ;$ minimum $(M i n)=7$, maximum $(\operatorname{Max})=20)$. While intelligence quotient (IQ) has shown to be unrelated to affordance perception (e.g. 
Block, 1993); to rule out this theoretical confound, an abridged version of the Wechsler Intelligence Scale for Children-IV (Vocabulary and Matrix Reasoning; Wechsler, 2003) was administered to ASD participants to ensure matched IQ with the community-recruited TD sample. The chosen short form configuration was selected because of its demonstrated high reliability and validity coefficients (Sattler \& Dumont, 2004) and rapid administration time (Ryan, Glass, \& Brown, 2007). IQ was obtained for all but two participants (analyses excluding these participants did not alter the pattern of results) and demonstrated average to above-average intellectual ability in the sample $(M=117.09, S D=8.35$; $M i n=106$, Max = 126). Additionally, we supplemented the diagnosis using strict cutoffs on the parent-report Social Communication Questionnaire (SCQ; Rutter, Bailey, \& Lord, 2005), a parent-report measure of the lifetime presence of a child's autistic symptoms that correlates highly with "gold standard" diagnostic measures (such as the ADOS), especially in those older than primary school age (Corsello et al., 2007). The SCQ is composed of three subscales: communicative ability, reciprocal social interaction, and restrictive/repetitive behaviors. A high SCQ score indicates more autistic symptoms.

Stimuli and apparatus. Participants were seated at white tables devoid of visual landmarks or texture gradients. For the graspability task, 18 square blocks of foam board $(1.25-\mathrm{cm}$ thick) were constructed, which ranged from $4 \mathrm{~cm}$ to $24 \mathrm{~cm}$ in width $(4,5,6,7,8,9,10,11,12$, $13,14,15,16,17,19,21,23$, and $24 \mathrm{~cm})$. Each block was marked with two parallel black lines, vertically placed along the edge on opposite sides of each block. These lines were 2 -cm long and $1 / 4$ of a $\mathrm{cm}$ thick. The lines served as reference points that indicated where the participants were to anticipate grasping the block. For the aperture task, a diamond-shaped hole was created between two pieces of foam board; the bottom foam board piece was attached to a wooden stand (see Figure 1). The size of the hole was manipulated by moving the top piece of foamboard upwards to increase hole size or downwards to decrease hole size. Paper rulers were affixed to the back of the poster board so that they framed the diamond shaped hole and indicated varying widths of the hole as it was adjusted to different sizes.

\section{Procedure}

Each participant completed all three action prediction tasks. Order of task was counter-balanced. For the reachability task, the experimenter moved a white plastic chip from the opposite side of the table toward the participants or away from a point directly in front of the participant to the opposite side of the table. The participants informed the experimenter when they thought the chip was at a location where it was just at the limit of their reach with their dominant hand without moving their shoulders from the back of the chair. Participants were encouraged to instruct the experimenter to make adjustments to the position of the chip. Participants made reachability estimates when the chip was moving toward or away from one of three locations-central (defined as $0^{\circ}$ starting from approximately $1.2 \mathrm{~m}$ away from the participant's midline and moving toward the participant), contralateral (starting $-30^{\circ}$ from center moving toward center, away from the reaching arm), and ipsilateral (starting $30^{\circ}$ from center plane moving toward the reaching arm) -for a total of six estimates (two estimates for each location; see Figure 1A). After participants made their estimation, they were asked to close their eyes, and using a measuring tape, the researcher measured the participant's estimate as defined by the distance between the chip and a dot positioned directly in front of participant on the edge of the table. After the researcher made the measurement, participants were instructed to open their eyes and the next trial began. The order of the estimates was randomized. At no time during the reaching-ability estimates were participants allowed to reach over the table. After participants estimated their reaching abilities, we assessed participants' actual reaching abilities for each arm in each direction.

For the graspability task, participants were presented with each of the blocks one at a time in random order. The blocks were positioned so that the two black lines on the blocks were in a vertical position with respect to the participant. Participants were told to anticipate whether they could grasp the block in a specific way. The way they were told to anticipate entailed placing their thumb on the black line on one edge of the block and then extending their hand across the entire block to place any one of their other fingers on the black line on the other side of the block, as overtly illustrated in Figure 1B. A successful grasp was defined as being able to lift the block completely off the table. After participants made their yes/no response, the present block was removed, and a new block was placed in front of the participants. The researcher made a point to hold the block at its corner across its height only using their index finger and thumb; this was done to prevent participants gaining feedback from the researcher's grasping abilities. At no point during the estimates were participants allowed to attempt grasping the blocks. After they finished all 18 estimates, the largest block that they could actually pick up was assessed.

In the aperture task, for two trials, subjects were presented with the largest sized hole, and the experimenter slowly decreased the size of the hole until participants indicated that they could just fit their dominant hand through the hole without touching the sides of the foamboard, as overtly illustrated in Figure 1C. For two other trials, participants made the same judgment except they 


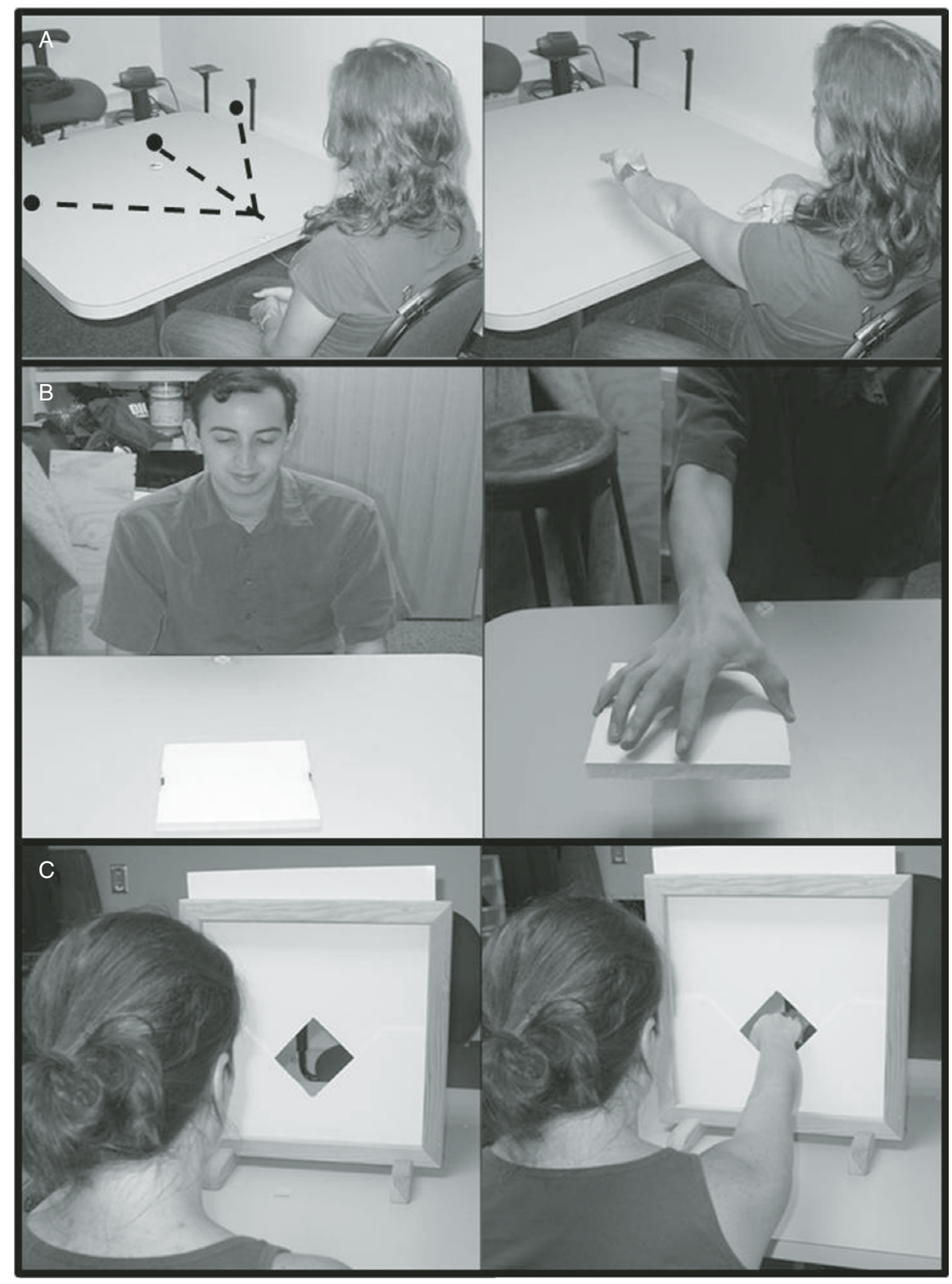

Figure 1. Visual illustrations of (A) the reachability task, (B) graspability task, and (C) aperture tasks.

were first presented with the smallest sized hole, and the experimenter slowly increased the size of the hole. After participants were satisfied that the hole's size was appropriate, the researcher assessed the hole size using the ruler on the back of the apparatus, and then the next trial began. Trial order was randomized. At no point during the estimates were participants allowed to reach through the aperture. Following the estimates, the smallest aperture that participants could fit their hand through was assessed.

All individuals understood the three tasks as evidenced in their successful performance of the actions overtly in the absence of further instructions. All measurements of anticipated and actual action capabilities were recorded on a data sheet.

\section{Results}

Percentage error was obtained for each subject on each task by taking the absolute value of the ratio of estimated ability (EA) over actual ability (AA) subtracted from 1 and multiplying by $100((1-(\mathrm{EA} / \mathrm{AA})) / 100)$. This is the percent amount of error deviation from AA in each estimate regardless of the direction of the error; thus, observed errors represented absolute deviations in either direction, not a general tendency toward over- or underestimation 

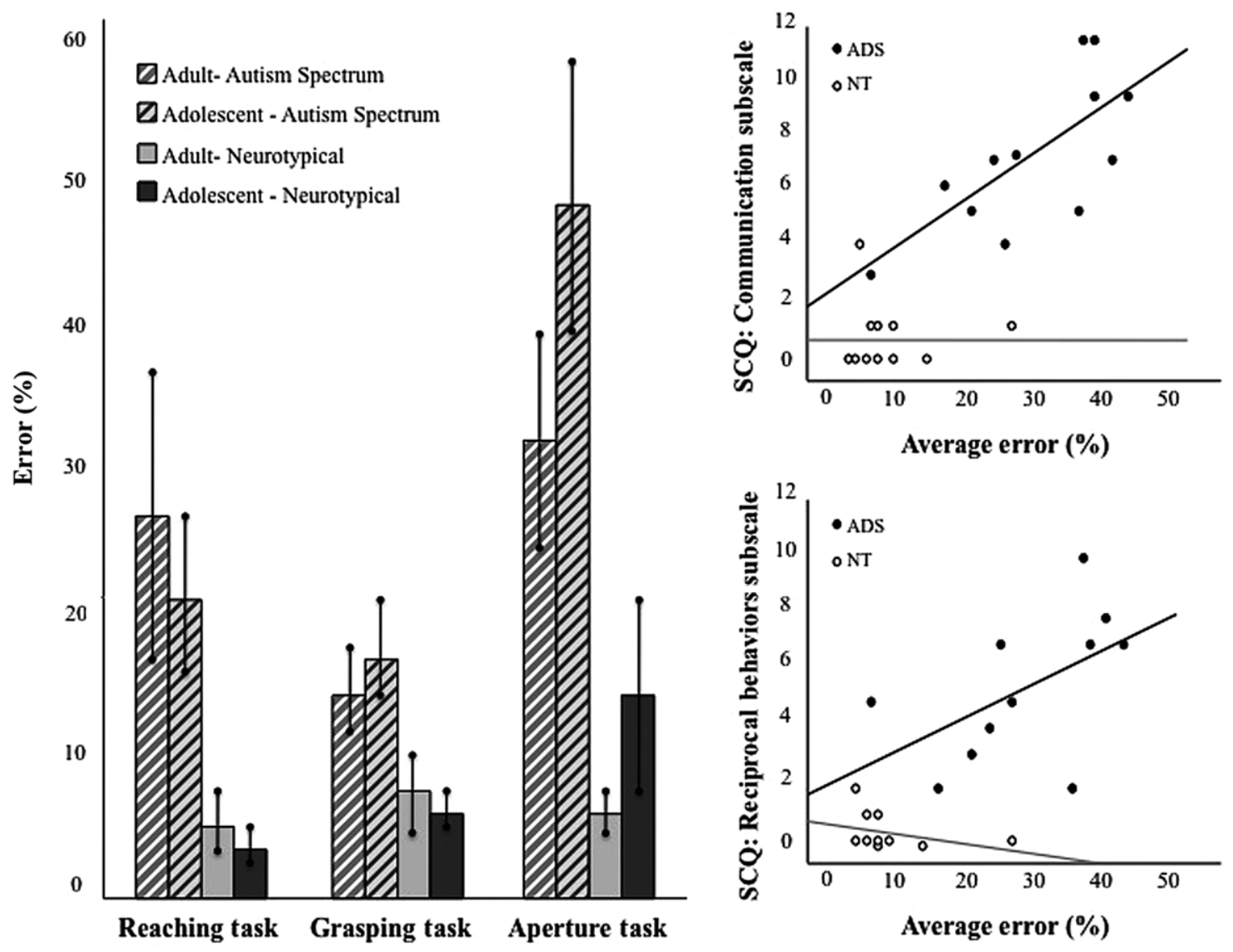

Figure 2. Left panel: The difference in performance of action capability estimation between autism spectrum disorder (ASD) and typically developing individuals. Error bars indicate \pm 1 standard error of the mean Right panel: The degree of error in such estimation was correlated with reported symptoms of communication and social deficits in the ASD population only.

(an analysis of over- and underestimation tendency can be seen in the Supplementary Materials). This error measure was used as the dependent measure in repeatedmeasures analysis of variance (ANOVA) with task as a within-subjects variable and ASD as between-subjects variable. There was a significant effect of task with aperture errors $[M=32 \%$, standard error $(S E)=6 \%]$ being larger than reachability $(M=13 \%, S E=3 \%)$ and grasping ability errors $(M=12 \%, S E=2 \%), F(2,44)=7.61, P<$ $0.01, \eta_{p}{ }^{2}=.26$. There were no significant interactions. As hypothesized, the amount of error in the ASD group significantly differed from the errors in the TD group, with adolescents with ASDs making drastically larger errors $(M=30 \%, S E=3 \%)$ than TD adolescents $(M=9 \%$, $S E=3 \%), \quad F(1,22)=30.82, \quad P<0.001, \quad \eta_{p}{ }^{2}=0.58 \quad$ (see Figure 2, left panel).

Notably, there was no significant univariate relationship between IQ and average error $(r=-0.48, P=0.16$, two-tailed test). However, severity of ASD social/ communicative symptoms predicted performance error on the action anticipation tasks. Higher SCQ scores predicted larger errors on the action anticipation tasks for
ASD participants, $r=0.58, P=0.02$, (all one-tailed tests), but not control participants, $P=0.26$. This correlation was driven by communication, $r=0.79, P=0.001$, and reciprocal social interaction, $r=0.55, P=0.03$, subscales only (see Figure 2, right panel). Additionally, IQ did not moderate the relationship between average error and total SCQ scores $(P=0.95)$ or any SCQ subscale (all $P>0.66)$.

\section{Experiment 2: Affordance Perception and Adults With ASDs}

Experiment 1 showed that adolescents with ASDs have difficulty determining their affordances. However, it is possible that individuals with ASDs develop these abilities later in life than TD individuals, or that errors were unique to the original sample. Thus, if these results were replicated in a young adult population, it will lend further support to the hypothesis that an impairment in basic perceptual-motor integration underlies the central deficit from which the lifelong symptoms associated with 
ASDs originate. We tested young adults with ASDs in the same tasks as the adolescents to see if the perceptualmotor deficit extends into adulthood.

\section{Methods}

Participants. Eight male adults (age 18-34, $M_{\text {age }}=22.38$ ) with high-functioning ASDs and eight TD male adults (age 17-21, $M_{\text {age }}=18.75$ ) participated in the experiment after giving informed consent. None of the participants had any significant hearing loss, visual impairment, or major physical disability. Documented diagnoses for the adults with ASDs were provided by area clinicians. However, we note that we were unable to access IQ or ADOS scores for these participants.

Materials and procedure. The materials and procedure were the same as used in experiment 1 . All individuals understood the tasks as evidenced in their successful performance of the actions overtly in the absence of further instructions.

\section{Results}

As in experiment 1, percentage error was calculated for each task for each participant. Percentage error was used as the dependent measure in repeated-measures ANOVA with task as a within-subjects variable and ASD as between-subjects variable. The results were almost identical to what we found in experiment 1 , except no differences between task were found, $P=0.28$. This can likely be explained by a lack of power because of a smaller sample size as the means differences between the tasks followed a similar pattern as in experiment 1 (aperture, $M=19 \%, S E=4 \%$; reachability, $M=17 \%, S E=5 \%$; grasping ability, $M=11 \%, S E=2 \%)$. There were no significant interactions. Importantly, the errors in the ASD adult group significantly differed from the errors in the TD group with adults with ASDs making drastically larger errors $(M=25 \%, S E=4 \%)$ than TD adults $(M=6 \%$, $S E=4 \%), \quad F(1,14)=14.14, \quad P=0.002, \quad \eta_{p}{ }^{2}=0.50 \quad$ (see Figure 2). When collapsing across experiments, on average, ASD individuals' estimations deviated from their actual capabilities by $28 \%$, in comparison with $8 \%$ from controls.

\section{Discussion}

Studies have shown that individuals with ASD have deficits using their motor systems to simulate and understand others' actions (Théoret et al., 2005; Williams, Whiten, \& Singh, 2004). Our findings raise the possibility that action-understanding deficits could originate in these individuals' inability to use their motor system to simulate their own actions to determine their action capabilities, which could hinder their ability to use the same process to interpret others' actions (Lombardo \& BaronCohen, 2011; Ramenzoni et al., 2008a).

These findings are notable in view of past work on motor abilities in ASD populations. As children with ASDs are just as accurate in the motoric execution of their actions as TD children (Forti et al., 2011; Glazebrook et al., 2006), the motor issues that individuals with ASDs do display appear to result from problems with motor planning, which is consistent with having a primary affordance perception deficit. Dowell, Mahone, and Mostofsky (2009) proposed (but did not test the hypothesis) that impairments in praxis were likely due to impairments in spatial perception and perceptual-motor integration due to the inability to alleviate this deficit when controlling for basic motor skills and postural knowledge. Similarly, people with ASDs are worse at adjusting already preplanned movements (Nazarali, Glazebrook, \& Elliott, 2009). Interestingly, perceptual motor integration deficits have also been found for individuals with Developmental Coordination Disorder (DCD); however, the deficits mostly extend to only the actions in which the individuals have difficulty performing (Johnson \& Wade, 2007). Although DCD is often comorbid with ASDs, it is unlikely that these are related due to the notion that individuals with ASD do not have deficits in performing the overt actions. Taken together, these findings suggest that our results were not likely due to impairments in the ability to execute a movement but rather the inability to integrate the information associated with planning the movement.

Our findings are especially striking in light of previous research suggesting that individuals with ASDs are actually superior to TD individuals in estimation tasks including those involving vision (Ashwin et al., 2009; Souliéres et al., 2010) and proprioceptive learning (Haswell et al., 2009; Mottron et al., 2006). It thus seems that the deficit does not stem from the individual sensory systems but the ability to integrate perceptual and motor information.

These results uncover a new perceptual-motor symptom associated with ASDs. Like other perceptual deficits in ASD populations (e.g. Klin, Lin, Gorrindo, Ramsay, \& Jones, 2009), this deficit occurs in a domain that usually develops fluidly at an extremely early age, not only in humans but also across the animal kingdom (Adolph \& Berger, 2006). That this deficit was found in adolescents and even adults, who might otherwise be expected to develop compensatory capacities, provides a strict test of the hypothesis that basic perceptual-motor capabilities are impaired in individuals with ASDs.

While these findings demonstrate the presence of perceptual-motor integration deficits in ASD, recent work in postural control in ASD suggest that it might be pre- 
served in some cases. Chang, Wade, Stoffregen, Hsu, and Pan (2010) found that children with ASD displayed a reduction in postural sway for visual tasks that require larger perceptual effort. Sway reduction with increased cognitive demands, a finding amply documented in TD adults, arises from the successful integration of postural control and cognitive task performance (e.g. Stoffregen, Pagulayan, Bardy, \& Hettinger, 2000). Chang et al.'s (2010) results suggest that ASD does not interfere with the ability to integrate perceptual and motor information during online performance. However, as the authors also suggests, this does not preclude difficulties in regulating sway for more complex tasks, such as holding a conversation or, as the present study demonstrates, the perception of action boundaries for future actions.

Importantly, affordance perception has shown to be quite plastic and susceptible to learning effects (Adolph, 1997; Fajen, 2005; Higuchi, Takada, Matsuura, \& Imanaka, 2004; Yu, Bardy, \& Stoffregen, 2010). Thus, there is reason to believe that the deficit revealed here may be amenable to intervention. That this deficit was found to so strongly relate to core deficits in ASDs suggests that such interventions may provide a simple, fruitful, and novel route to treating complex social/ communicative problems in this population.

There are several factors limiting interpretation of these results that bear mention. First, while previous research has shown that IQ does not affect performance on motor planning (Robinson, Goddard, Dritschel, Wisley, \& Howlin, 2009) or affordance perception (Block, 1993) tasks, reliance on a high-functioning sample limits the ability to generalize these findings to individuals with ASD and poorer IQ (i.e. such individuals may experience the deficit revealed here, as well as additional deficits associated with poor task understanding). Similarly, the lack of formal IQ or ASD symptomology (e.g. SCQ) assessment in the adult sample limits the ability to generalize from that sample. Therefore, the results from the adult sample should be seen primarily as a replication of the findings from the adolescent sample using a community clinician-diagnosed, slightly older sample, and not strong verification of the identified phenomenon in older individuals. Second, the correlational data do not permit evaluation of direction of effects. As such, while the present interpretation (that social deficits may emerge from affordance perception deficits) is consonant with current research specifying basic perceptual deficits as underlying subsequent complex social deficits (Klin, Jones, Schultz, \& Volkmar, 2003; Klin et al., 2009; McPartland et al., 2011), these results cannot rule out the possibility that the direction of the observed effects is reversed (especially given participants' ages). Indeed, just as poor theory of mind might be intertwined with poor self-reflection among individuals with ASDs (Lombardo \& Baron-Cohen,
2011), social difficulties may be reciprocally related to (and possibly upstream of) affordance perception. Finally, it is possible that extraneous factors (e.g. a desire to complete the task quickly, a perfectionistic tendency, or interpretation of experimenters' social nuance) could have influenced both execution speed and performance. While the design featured unlimited estimation and correction time, past research suggests that speed of completion does not affect performance on these tasks (Heft, 1993). As a result, differences in speed of completion likely did not influence observed between-group differences. Yet future research would do well to consider the role of such factors in explaining deficits in affordance perception in this population.

Optical information specifying spatial layout comes to the eye in terms of visual angles. When perceiving extents, perceivers scale optical information to their bodies using their action capabilities as perceptual rulers (Proffitt, 2008). For example, the distances to reachable objects are seen as a proportion of the perceiver's maximum reach (Linkenauger, Witt, Stefanucci, Bakdash, \& Proffitt, 2009; Witt, Proffitt, \& Epstein, 2005). Thus, individuals do not perceive the environment in isolation, but they perceive it in relation to their bodies. The present study found that ASD populations do not readily achieve this perceptual body scaling. Thus, such individuals may not perceive a precise fit between themselves and their perceptual world and, as a consequence, may find themselves perceptually isolated from it.

Perceiving affordances is fundamental to performing everyday tasks. Gross impairments in this ability could conceivably underlie more commonly known symptoms in ASDs, such as the ability to socialize and communicate. This possibility is raised by the relationship between social deficits and affordance perception deficits uncovered here. In this respect, these findings are important for the development of objective diagnostic criteria for ASDs that could complement the observational, report-based methods currently employed. Further efforts in this direction will lend theoretical support and augment the focus of therapeutic interventions. Specifically, training of perceptual-motor integration (specifically affordance perception, which is currently nonexistent in ASD treatment) could provide a novel avenue of treatment that may lead to the amelioration of putative core deficits.

\section{Acknowledgments}

Portions of this article were presented at the May 2011 Research Academy of the LIFE Program, Ann Arbor, MI. We would like to thank the children and parents who participated, as well as the colleagues who read previous drafts of this manuscript. All authors report no biomedical financial interests or potential conflicts of interest. 


\section{References}

Adolph, K.E. (1997). Learning in the development of infant locomotion. [Research Support, Non-U.S. Gov't Research Support, U.S. Gov't, P.H.S.]. Monographs of the Society for Research in Child Development, 62, I-VI, 1-158.

Adolph, K.E., \& Berger, S.E. (2006). Motor development. In W. Damon \& R. Lerner (Series Eds.) \& D. Kuhn \& R.S. Siegler (Vol. Eds.), Handbook of child psych, Vol. 2, 6th ed (pp. 161-213). Hoboken: John Wiley \& Sons.

Ashwin, E., Ashwin, C., Rhydderch, D., Howells, J., \& BaronCohen, S. (2009). Eagle-eyed visual acuity: An experimental investigation of enhanced perception in autism. Biological Psychiatry, 65, 17-21.

Asperger, H. (1994). Die Autistschen Psychopathen im Kindesalter. Archiv fuer Psychiatrie und Nerven Krankheiten, 117, 132-136.

Attwood, T. (2008). Complete guide to Asperger's syndrome. Philadelphia: Jessica Kingsley.

Baranek, G.T., Parham, L.D., \& Bodfish, J.W. (2005). Sensory and motor features in autism: Assessment and intervention. In F. Volkmar, A. Klin \& R. Paul (Eds.), Handbook of Autism and Pervasive Developmental Disorders: Vol. 2. Assessment, Interventions and Policy, 3rd ed. (pp. 831-857). Hoboken: Wiley $\&$ Sons.

Blake, R., Turner, L.M., Smoski, M.J., Pozdol, S.L., \& Stone, W.L. (2003). Visual recognition of biological motion is impaired in children with autism. Psychological Science, 14, 151-157. doi: 10.1111/1467-9280.01434

Blakemore, S.J., \& Decety, J. (2001). From the perception of action to the understanding of intention. Nature Reviews. Neuroscience, 2, 561-567.

Block, M.E. (1993). Can children with mild mental retardation perceive affordances for action? Adapted Physical Activity Quarterly, 10, 137-145.

Boker, S.M., Cohn, J.F., Theobald, B.-J., Matthews, I., Mangini, M., Spies, J.R., . . Brick, T.R. (2011). Something in the way we move: Motion dynamics, not perceived sex, influence head movements in conversation. Journal of Experimental Psychology: Human Perception and Performance, 37, 874891.

Boria, S., Fabbri-Destro, M., Cattaneo, L., Sparaci, L., Sinigaglia, C., Santelli, E., ... Rizzolatti, G. (2009). Intention understanding in autism. PLoS ONE, 4, e5596.

Carpenter, M., Pennington, B.F., \& Rogers, S.J. (2001). Understanding of others' intentions in children with autism. Journal of Autism and Developmental Disorders, 31, 589599. doi: 10.1023/a:1013251112392

Chang, C., Wade, M.G., \& Stoffregen, T.A. (2009). Perceiving affordances for aperture passage in an environment-personperson system. Journal of Motor Behavior, 41, 495-500. doi: 10.3200/35-08-095

Chang, C.-H., Wade, M.G., Stoffregen, T.A., Hsu, C.-Y., \& Pan, C.-Y. (2010). Visual tasks and postural sway in children with and without autism spectrum disorders. Research in Developmental Disabilities, 31, 1536-1542. doi:10.1016/ j.ridd.2010.06.003.

Cook, J., Saygin, A.P., Swain, R., \& Blakemore, S.-J. (2009). Reduced sensitivity to minimum-jerk biological motion in autism spectrum conditions. Neuropsychologia, 47, 32753278. doi: 10.1016/j.neuropsychologia.2009.07.010.

Corsello, C., Hus, V., Pickles, A., Risi, S., Cook, E.H., Jr., Leventhal, B.L., et al. (2007). Between a ROC and a hard place: Decision making and making decisions about using the SCQ. Journal of Child Psychology and Psychiatry, 48, 932-940.

Davis, T.J., Riley, M.A., Shockley, K., \& Cummins-Sebree, S. (2010). Perceiving affordances for joint actions. Perception, 39, 1624-1644. doi: 10.1068/p6712

de C. Hamilton, A.F., Brindley, R.M., \& Frith, U. (2007). Imitation and action understanding in autistic spectrum disorders: How valid is the hypothesis of a deficit in the mirror neuron system? Neuropsychologia, 45, 1859-1868. doi: 10.1016/ j.neuropsychologia.2006.11.022

de Marchena, A., \& Eigsti, I.-M. (2010). Conversational gestures in autism spectrum disorders: Asynchrony but not decreased frequency. Autism Research, 3, 311-322. doi:10.1002/ aur.159.

Dewey, D. (1995). What is developmental dyspraxia? Brain and Cognition, 29, 254-274. doi:10.1006/brcg.1995.1281.

Dewey, D., \& Kaplan, B.J. (1992). Analysis of praxis task demands in the assessment of children with developmental motor deficits. Developmental Neuropsychology, 8, 367-379. doi: 10.1080/87565649209540532

Dowell, L.R., Mahone, E.M., \& Mostofsky, S.H. (2009). Associations of postural knowledge and basic motor skill with dyspraxia in autism: Implication for abnormalities in distributed connectivity and motor learning. Neuropsychology, 23, 563570. doi: 10.1037/a0015640

Fabbri-Destro, M., \& Rizzolatti, G. (2008). Mirror neurons and mirror systems in monkeys and humans. Physiology (Bethesda), 23, 171-179.

Fajen, B.R. (2005). The scaling of information to action in visually guided braking. [Research Support, Non-U.S. Gov't]. Journal of Experimental Psychology. Human Perception and Performance, 31, 1107-1123. doi: 10.1037/00961523.31.5.1107

Falck-Ytter, T. (2010). Young children with autism spectrum disorder use predictive eye movements in action observation. Biology Letters, 6, 375-378.

Forti, S., Valli, A., Perego, P., Nobile, M., Crippa, A., \& Molteni, M. (2011). Motor planning and control in autism. A kinematic analysis of preschool children. Research in Autism Spectrum Disorders, 5, 834-842.

Freitag, C.M., Konrad, C., Häberlen, M., Kleser, C., von Gontard, A., Reith, W., ... Krick, C. (2008). Perception of biological motion in autism spectrum disorders. Neuropsychologia, 46, 1480-1494. doi: 10.1016/j.neuropsychologia.2007.12.025

Gibson, J.J. (1966). The senses considered as perceptual systems. Oxford, England: Houghton Mifflin.

Gibson, J.J. (1979). The ecological approach to visual perception. Boston, MA, USA: Houghton, Mifflin.

Glazebrook, C.M., Elliott, D., \& Lyons, J. (2006). A kinematic analysis of how young adults with and without autism plan and control goal-directed movements. Motor Control, 10, 244-264.

Haswell, C.C., Izawa, J., Dowell, L.R., Mostofsky, S.H., \& Shadmehr, R. (2009). Representation of internal models of action in the autistic brain. Nature Neuroscience, 12, 970-972. 
Heft, H. (1993). A methodological note on overestimates of reaching distance: Distinguishing between perceptual and analytical judgments. Ecological Psychology, 5, 255-271. doi: 10.1207/s15326969eco0503_3

Higuchi, T., Takada, H., Matsuura, Y., \& Imanaka, K. (2004). Visual estimation of spatial requirements for locomotion in novice wheelchair users. Journal of Experimental Psychology, 10, 55-66. doi: 10.1037/1076-898x.10.1.55

Hubert, B., Wicker, B., Moore, D.G., Monfardini, E., Duverger, H., et al. (2007). Brief report: Recognition of emotional and non-emotional biological motion in individuals with autistic spectrum disorders. Journal of Autism and Developmental Disorders, 37, 1386-1392. doi: 10.1007/s10803-006-0275-y

Ishak, S., Adolph, K.E., \& Lin, G.C. (2008). Perceiving affordances for fitting through apertures. Journal of Experimental Psychology: Human Perception and Performance, 34, 15011514.

Johnson, D.C., \& Wade, M.G. (2007). Judgment of action capabilities in children at risk for developmental coordination disorder. Disability and Rehabilitation: An International, Multidisciplinary Journal, 29, 33-45. doi: 10.1080/ 09638280600947708

Kaiser, M.D., \& Shiffrar, M. (2009). The visual perception of motion by observers with autism spectrum disorders: A review and synthesis. Psychonomic Bulletin and Review, 16, 761-777. doi:10.3758/pbr.16.5.761.

Klimkeit, E.I., Mattingley, J.B., Sheppard, D.M., Lee, P., \& Bradshaw, J.L. (2005). Motor preparation, motor execution, attention, and executive functions in Attention Deficit/ Hyperactivity Disorder (ADHD). Child Neuropsychology, 11, 153-173.

Klin, A., Jones, W., Schultz, R., \& Volkmar, F. (2003). The enactive mind, or from actions to cognition: Lessons from autism. Philosophical Transactions of the Royal Society of London. Series B, Biological Sciences, 358, 345-360. doi: 10.1098/ rstb.2002.1202

Klin, A., Lin, D., Gorrindo, P., Ramsay, G., \& Jones, W. (2009). Two-year-olds with autism orient to non-social contingencies rather than biological motion. Nature, 459, 257-261.

Koldewyn, K., Whitney, D., \& Rivera, S.M. (2011). Neural correlates of coherent and biological motion perception in autism. Developmental Science, 14, 1075-1088. doi: 10.1111/j.14677687.2011.01058.x

Konczak, J., Meeuwsen, H.J., \& Cress, M.E. (1992). Changing affordances in stair climbing: The perception of maximum climbability in young and older adults. Journal of Experimental Psychology: Human Perception and Performance, 18, 691-697. doi: 10.1037/0096-1523.18.3.691

Linkenauger, S.A., Witt, J.K., Stefanucci, J.K., Bakdash, J.Z., \& Proffitt, D.R. (2009). The effects of handedness and reachability on perceived distance. Journal of Experimental Psychology: Human Perception and Performance, 35, 16491660.

Lombardo, M.V., \& Baron-Cohen, S. (2011). The role of the self in mindblindness in autism. Consciousness and Cognition: An International Journal, 20, 130-140.

Lord, C., Rutter, M., DiLavore, P., \& Risi, S. (1999). Manual for the autism diagnostic observation schedule. Los Angeles, CA: Western Psychological Services.
Mark, L.S. (1987). Eyeheight-scaled information about affordances: A study of sitting and stair climbing. Journal of Experimental Psychology: Human Perception and Performance, 13, 361-370. doi: 10.1037/0096-1523.13.3.361

Mark, L.S., Balliett, J.A., Craver, K.D., Douglas, S.D., et al. (1990). What an actor must do in order to perceive the affordance for sitting. Ecological Psychology, 2, 325-366. doi: 10.1207/ s15326969eco0204_2

Marsh, K.L., Richardson, M.J., \& Schmidt, R.C. (2009). Social connection through joint action and interpersonal coordination. Topics in Cognitive Science, 1, 320-339.

McKay, L.S., Simmons, D.R., McAleer, P., Marjoram, D., Piggot, J., \& Pollick, F.E. (2012). Do distinct atypical cortical networks process biological motion information in adults with Autism Spectrum Disorders? Neuroimage, 59, 1524-1533. doi: 10.1016/j.neuroimage.2011.08.033

McPartland, J.C., Wu, J., Bailey, C.A., Mayes, L.C., Schultz, R.T., \& Klin, A. (2011). Atypical neural specialization for social percepts in autism spectrum disorder. Society for Neuroscience, 6, 436-451.

Ming, X., Brimacombe, M., \& Wagner, G.C. (2007). Prevalence of motor impairment in autism spectrum disorders. Brain and Development, 29, 565-570.

Moore, D.G., Hobson, R.P., \& Lee, A. (1997). Components of person perception: An investigation with autistic, nonautistic retarded and typically developing children and adolescents. British Journal of Developmental Psychology, 15, 401-423. doi: 10.1111/j.2044-835X.1997.tb00738.x

Mottron, L., Dawson, M., Souliéres, I., Hubert, B., \& Burack, J. (2006). Enhanced perceptual functioning in autism: An update, and eight principles of autistic perception. Journal of Autism and Developmental Disorders, 36, 27-43.

Murphy, P., Brady, N., Fitzgerald, M., \& Troje, N.F. (2009). No evidence for impaired perception of biological motion in adults with autistic spectrum disorders. Neuropsychologia, 47, 3225-3235. doi: 10.1016/j.neuropsychologia.2009. 07.026

Nazarali, N., Glazebrook, C.M., \& Elliott, D. (2009). Movement planning and reprogramming in individuals with autism. Journal of Autism and Developmental Disorders, 39, 14011411.

Oudejans, R.R.D., Michaels, C.F., Bakker, F.C., \& Dolné, M.A. (1996). The relevance of action in perceiving affordances: Perception of catchableness of fly balls. Journal of Experimental Psychology: Human Perception and Performance, 22, 879-891. doi: 10.1037/0096-1523.22.4.879

Parkinson, J., Springer, A., \& Prinz, W. (2011). Can you see me in the snow? Action simulation aids the detection of visually degraded human motion. The Quarterly Journal of Experimental Psychology, 64, 1463-1472. doi: 10.1080/ 17470218.2011.594895

Price, K.J., Shiffrar, M., \& Kerns, K.A. (2012). Movement perception and movement production in Asperger's Syndrome. Research in Autism Spectrum Disorders, 6, 391398.

Proffitt, D.R. (2008). An action-specific approach to spatial perception. In R.L. Klatzky, B. MacWhinney \& M. Behrmann (Eds.), Embodiment, ego-space, and action (pp. 179-202). New York: Psychology Press. 
Ramenzoni, V.C., Davis, T.J., Riley, M.A., Shockley, K., \& Baker, A.A. (2011). Joint action in a cooperative precision task: Nested processes of intrapersonal and interpersonal coordination. Experimental Brain Research, 211, 447-457.

Ramenzoni, V.C., Riley, M.A., Shockley, K., \& Davis, T. (2008a). Carrying the height of the world on your ankles: Encumbering observers reduces estimates of how high an actor can jump. Quarterly Journal of Experimental Psychology, 61, 1487-1495.

Ramenzoni, V.C., Riley, M.A., Shockley, K., \& Davis, T. (2008b). An information-based approach to action understanding. Cognition, 106, 1059-1070. doi: 10.1016/j.cognition.2007. 04.008

Richardson, M.J., Marsh, K.L., \& Baron, R.M. (2007). Judging and actualizing intrapersonal and interpersonal affordances. Journal of Experimental Psychology: Human Perception and Performance, 33, 845-859. doi: 10.1037/0096-1523.33.4.845

Robinson, S., Goddard, L., Dritschel, B., Wisley, M., \& Howlin, P. (2009). Executive functions in children with autism spectrum disorders. Brain and Cognition, 71, 362-368.

Rondan, C., \& Deruelle, C. (2005). Developmental trends in visuo-spatial abilities in the autistic pathology. Cahiers de Psychologie Cognitive/Current Psychology of Cognition, 23, 198-204.

Rutter, M., Bailey, A., \& Lord, C. (2005). SCQ: The social communication questionnaire manual. Los Angeles: Western Psychological Services.

Ryan, J.J., Glass, L.A., \& Brown, C.N. (2007). Administration time estimates for Wechsler Intelligence Scale for Children-IV subtests, composites, and short forms. Journal of Clinical Psychology, 63, 309-318.

Sattler, J.M., \& Dumont, R. (2004). Assessment of children: WISC-IV and WPPSI-III supplement. San Diego, CA: J.M. Sattler.

Simion, F., Regolin, L., \& Bulf, H. (2008). A predisposition for biological motion in the newborn baby. Proceedings of the National Academy of Sciences of the United States of America, 105, 809-813. doi: 10.1073/pnas.0707021105

Sinzig, J., Walter, D., \& Doepfner, M. (2009). Attention deficit/ hyperactivity disorder in children and adolescents with autism spectrum disorder: Symptom or syndrome? Journal of Attention Disorders, 13, 117-126.

Souliéres, I., Hubert, B., Rouleau, N., Gagnon, L., Tremblay, P., et al. (2010). Superior estimation abilities in two autistic spectrum children. Cognitive Neuropsychology, 27, 261276.

Springer, A., Brandstädter, S., Liepelt, R., Birngruber, T., Giese, M., et al. (2011). Motor execution affects action prediction. Brain and Cognition, 76, 26-36. doi: 10.1016/j.bandc.2011. 03.007

Stoffregen, T.A., Pagulayan, R.J., Bardy, B.B., \& Hettinger, L.J. (2000). Modulating postural control to facilitate visual performance. Human Movement Science, 19, 203-220. doi: 10.1016/s0167-9457(00)00009-9

Stoffregen, T.A., Yang, C.-M., \& Bardy, B.G. (2005). Affordance judgments and nonlocomotor body movement. Ecological Psychology, 17, 75-104. doi: 10.1207/s15326969eco1702_2
Théoret, H., Halligan, E., Kobayashi, M., Fregni, F., TagerFlusberg, H., \& Pascual-Leone, A. (2005). Impaired motor facilitation during action observation in individuals with autism spectrum disorder. Current Biology, 15, R84-R85.

Warren, W.H. (1984). Perceiving affordances: Visual guidance of stair climbing. Journal of Experimental Psychology: Human Perception and Performance, 10, 683-703. doi: 10.1037/ 0096-1523.10.5.683

Warren, W.H., \& Whang, S. (1987). Visual guidance of walking through apertures: Body-scaled information for affordances. Journal of Experimental Psychology: Human Perception and Performance, 13, 371-383.

Wechsler, D. (2003). Manual for the Wechsler intelligence scale for children-Fourth edition (WISC-IV). New York: Psychological Corporation/Harcourt Brace.

Whyatt, C., \& Craig, C. (in press). Motor skills in children aged 7-10 years, diagnosed with autism spectrum disorder. Journal of Autism and Developmental Disorders, 1-11. doi: 10.1007/ s10803-011-1421-8

Williams, J.H.G., Whiten, A., \& Singh, T. (2004). A systematic review of action imitation in autistic spectrum disorder. Journal of Autism and Developmental Disorders, 34, 285 299.

Wing, L. (1981). Asperger's syndrome; a clinical account. Psychological Medicine, 11, 115-130.

Witt, J.K., Proffitt, D.R., \& Epstein, W. (2005). Tool use affects perceived distance, but only when you intend to use it. Journal of Experimental Psychology: Human Perception and Performance, 31, 880-888.

Yu, Y., Bardy, B.G., \& Stoffregen, T.A. (2010). Influences of head and torso movement before and during affordance perception. Journal of Motor Behavior, 43, 45-54. doi: 10.1080/ 00222895.2010 .533213

Zalla, T., Labruyere, N., Clement, A., \& Georgieff, N. (2010). Predicting ensuing actions in children and adolescents with autism spectrum disorders. Exp Brain Res, 201, 809-819.

\section{Supporting Information}

Additional Supporting Information may be found in the online version of this article:

Figure S1. Ratios of estimated/actual for the difference conditions across the three tasks. Error bars represent +/1 SE of the mean.

Table S1. Means and SE for the estimated/action values in the different action anticipant tasks across the different populations.

Please note: Wiley-Blackwell are not responsible for the content or functionality of any supporting materials supplied by the authors. Any queries (other than missing material) should be directed to the corresponding author for the article. 